\title{
Ultrastructural Studies on the Anal Canal Epithelium in the Mouse, with Special Reference to the Keratinizing Process
}

\author{
By \\ T. NAKANO \\ Department of Anatomy, Aichi Medical University, Nagakute, Aichi 480-11, Japan \\ - Received for Publication, July 9, 1996-
}

Key Words: Anal canal, Epithelium, Microridge, Keratinization

\begin{abstract}
Summary: The stratified squamous epithelium lining the mouse anal canal revealed various stages of keratinization. Considering the ultrastructural features, it is suggested that the epithelium lining the distal part shows more advanced keratinization than in the proximal part. Further, the width of the microridges in the distal part was about doubled compared to that in the proximal part. The difference in the width of microridges appears to be an important marker of the degree of keratinization.
\end{abstract}

The term "anal canal" was first used by Symington (1888), who indicated that the anal canal extended from the anorectal ring to the anal opening. This definition corresponds to the surgical anal canal, while the term anatomical anal canal has been used for the part between the pectinate line and the anal margin. In the present study, the anal canal is defined as the duct extending from the pectinate line to the anal margin, considering not only the anatomical but the embryological findings (Nakano and Muto, 1987). The stratified squamous epithelium lining the anal canal occupies the transitional zone between the mucosal epithelium in the rectum and the perianal epidermis, and shows the concomitant presence of features denoting similarities to, and differences from, the epidermis. The epithelium has been assigned a variety of names such as the "anoderm" (Takano, 1978), "modified skin" (Goligher et al., 1955), "transitional skin" (Baba, 1968) and "specialized anal canal skin" (Henrich, 1980).

Whether the anal canal epithelium is keratinized or not has been the subject of investigation. Some investigators reported in man that the epithelium was nonkeratinized (Henrich, 1980; Fenger and Knoth, 1981; Fujita and Fujita, 1992; Fenger, 1992). On the other hand, it was reported that the distal part of the anal canal was keratinized but the proximal part was not in man (Johnson, 1914) and rhesus monkey (Jit, 1974). Further, Klotz et al. (1967) mentioned that the human anal canal epithelium was keratinized up to the pectinate line. It is difficult to conclude whether a mucosal epithelium is keratinized or not, one reason being that the term "keratin- ization" has acquired somewhat different meanings according to the viewpoint and interest of the observers. It is the purpose of this paper to present a dependable marker of keratinization, because the anal canal epithelium is expected to demonstrate advantageously the various stages in the keratinizing process.

\section{Material and Methods}

The mucosal membrane in the anorectal region was removed from $20 \mathrm{ddN}$ mice $(10$ male and 10 female) under a dissecting microscope. Transmission electron microscopic specimens were sliced into blocks, immediately fixed for 3 hours in $2.5 \%$ glutaraldehyde in phosphate buffer, and postfixed for 2 hours in $2 \%$ osmium tetroxide. The specimens were dehydrated in ascending concentrations of ethanol, transferred to propylene oxide and embedded in Epon 812. Ultrathin sections were cut with a ultramicrotome and double-stained with uranyl acetate and lead citrate. They were examined with a Hitachi HU-12A transmission electron microscope.

Scanning electron microscopic samples were fixed in $2.5 \%$ glutaraldehyde and dehydrated in graded ethanol. After immersion in 1\% tannic acid for 2-3 hours, they were postfixed with $2 \%$ osmium tetroxide for 1 hour, dehydrated in a graded ethanol, and dried with t-butyl alcohol freeze-drying method (Inoue and Osatake, 1988). The specimens were then coated with gold and observed under the Jeol 
JSM 6100 scanning electron microscope.

\section{Results}

\section{Transmission electron-microscopic findings}

In the proximal part of the anal canal, many keratohyalin granules were observed in the granular layer. They were round or oval in shape, and had homogeneous contents of high electron density, sometimes a central spot of low density. All the keratohyalin granules were surrounded by many ribosomes but did not appear to have any relation to the filaments (Fig. 1, 2). The superficial layer had several plates of flattened cells from which intact nuclei, mitochondria and other organelles had disappeared. In the cytoplasm, the individual filaments were distinctly seen and showed some tendency to form fibrils (Fig. 1).

In the middle part, keratohyalin granules were found in cells of the granular layer. Some granules consisted of homogeneous contents of high electron density (Fig. 1). Other granules were composed of a dense rim, surrounding material which formed configurations of different density. These granules were occasionally associated with filaments (Fig. 3). The cytoplasm of the superficial cells was filled with opaque filaments which were embedded in a less opaque interfilamentous substance. The microridges were thicker than those in the proximal part (Fig. 1).

In the distal part of the anal canal, the cells in the granular layer contained a fairly large number of irregularly shaped keratohyalin granules (Fig. 4). Some granules had a mottled appearance (Fig. 5a), while others were masses of homogeneous content with high electron density (Fig. 5b). These granules were associated with filaments (Fig. 4, 5). In the cytoplasm of the superficial cell, opaque filaments were embedded in a less opaque interfilamentous substance. The individual filaments were not always clearly seen (Fig. 4). The cytoplasmic membrane of the superficial cells consisted of an asymmetrical trilamellar structure which was thicker and denser than that in the superficial cells in the proximal part. This thickening was made by an electron-dense inner leaflet of the membrane (Fig. 4). The microridges were not clearly seen.

\section{Scanning electron miçroscopic findings}

The anal canal epithelium showed a typical surface appearance of stratified squamous epithelium; i.e., the superficial cells appeared to be relatively flat and arranged in a paving-stone appearance (Fig. 6). Higher magnification revealed that the surface was different according to the region of location. In the proximal part, the exposed surfaces of individual superficial cells had microridges about $0.1-0.2 \mu \mathrm{m}$ in width (Fig. 7). Although the microridges were occasionally arranged parallel to the cell boundaries in the periphery of the cell, there was no specific pattern of arrangement. In the middle part, microridges were approximately $0.3-0.4 \mu \mathrm{m}$ in width, about twice the size of those in the proximal part (Fig. 7). As the surface was traced distally, the microridges tended to coalesce and so the outlines became indistinct (Fig. 8). Near the anal margin, the surface showed a relatively smooth appearance (Fig. 9).

\section{Discussion}

What is a dependable marker of keratinization? At least, there is no doubt about the presence of keratinization in normal epidermis. Brody (1960) observed the "keratin pattern" with less opaque filaments embedded in an opaque interfilamentous substance in the cornified layer of human epidermis. That is, the mucosa representing the keratin pattern is keratinized. In some areas of the mucosa, such as the lingual filiform papilla of rat (Farbman, 1970) and the penile papilla of the rat glans penis (Patrizi and Munger, 1966), the keratin pattern was observed. In most areas of the mucosa, however, the keratin pattern was not observed, and the cells in the cornified layer had their opaque filaments embedded in a less opaque interfilamentous substance. This is known as the "abnormal keratin pattern". According to McMillan (1979), examination in LM of sections stained with Mallory's triple connective-tissue stain revealed that the rat hard palate which exhibited the keratin pattern in TEM showed complete keratinization, while the rat cheek which exhibited the abnormal keratin pattern displayed incomplete keratinization. Thus, the mucosal keratinization is included within a broad continuous spectrum ranging from complete to incomplete keratinization. In other words, there is no dependable marker of mucosal keratinization, making it difficult to conclude whether a mucosa is keratinized or not.

The anal canal extends from the pectinate line to the anal margin. At the pectinate line the stratified squamous epithelium lining the anal canal changes into the simple columnar epithelium lining the rectum, while distally the epithelium continues into the epidermis lining the anal margin (Henrich, 1980; Nakano and Muto, 1987). In other words, the epithelium lining the anal canal occupies the transitional zone between the "nonkeratinized" simple columnar epithelium and the "highly keratinized" epidermis, and is expected to show various stages in the differentiation of the epithelium towards keratinization. 
Hence, when the epithelium is traced from the pectinate line to the anal margin, a dependable marker of the start of keratinization may be revealed.

According to Rothman (1954), keratohyalin granules appeared in a nonkeratinized epithelium as soon as the epithelium acquired the potentiality of keratinization. Nakano (1986) observed various types of keratohyalin granules in the "intermediate epithelium" which occupied the transitional zone between the ciliated columnar epithelium and the keratinized stratified squamous one in the mouse nasopharynx. The intermediate epithelium represented earlier stages of keratinization. Further, Nakano (1986) classified the keratohyalin granules into 5 types (K1-5) considering not only their morphological differences but their developmental stages, and pointed out that different morphological types of the granule were associated with differences in the degree of keratinization. In the present study, the thickening of the cytoplasmic membrane and the opacity of cytoplasm, both of which were characteristic features of the keratinized epithelium, were more distinct in the distal part of the anal canal than in the proximal one. On the basis of these findings, it is suggested that the epithelium lining the distal part shows more advanced keratinization than that in the proximal part. In the proximal part, the keratohyalin granules were oval or round in shape, and surrounded by many ribosomes; the impression is that the granules are derived from ribosomes, which become progressively more condensed until their morphology changes from a granular to an amorphous form. Considered from their morphological features, these granules appear to correspond to the $\mathrm{K} 1-\mathrm{K} 3$ type granules (Nakano, 1986) or the immature granules (Muto, 1970). In the distal part, the keratohyalin granules were irregularly shaped and were associated with filaments. These granules appear to correspond to the mature granules (Muto, 1970), and to show an ultimate stage in the development of keratohyalin granules. These findings may lend support to the earlier report that in the epithelium showing the abnormal keratin pattern the keratohyalin granules were regularly shaped and not associated with filaments, whereas in the epithelium showing the keratin pattern contained irregularly shaped keratohyalin granules associated with filaments (McMillan, 1979). However, this is not always the case because the posterior surface of the lingual filiform papilla of rat and cow, which lacks keratohyalin granules, has the keratin pattern (Farbman, 1970). Further, in the middle part of the anal canal, the relationship between the morphological types of keratohyalin granule and the degree of keratinization was more ambiguous; i.e., the keratohyalin granules had 2 different components with various configurations. They are similar morphologically to the K4 type granules (Nakano, 1986). That is, different morphological types of keratohyalin granule can not be regarded as a dependable marker of the degree of keratinization.

With SEM, the term keratinization has become even more ambiguous. Nonkeratinized epithelium had microridges on its exposed surface. It is considered that microridges are only the remains of usual cytoplasmic processes which were once necessary to maintain intercellular organization (Nakano, 1986). On the other hand, the honeycomb appearance formed by interconnections of microridges surrounding depressions was regarded as a characteristic on the exposed surface of keratinized epithelium in general (Cleaton-Jones, 1975; Nair and Rossinsky, 1984). In the areas where keratinization was more advanced, microridges cohered to one another to give a relatively smooth appearance (Nakano, 1986, 1996; Nakano and Muto, 1986). In other words, microridges disappear when the keratinization increases beyond a certain degree. However, this is not always the case because parts of the glans penis of the mouse, which was keratinized, had microridges (Nakano, 1996). Thus, microridges are observed on the exposed surface not only in the nonkeratinized epithelium but in the keratinized one. Nakano (1986) reported at first that intercellular processes and microridges in epithelium representing a more advanced keratinization were wider than those in the epithelium showing less advanced keratinization, and suggested that the intercellular processes increased in size as a result of cell membrane distortion associated with keratinization, to form the tightly applied intercellular interdigitations as the cells moved towards the cornified layer.

In this study, the surface of the epithelium lining the anal canal was covered by various microridge patterns. The width of the microridges in the middle part showing more advanced keratinization was about double that in the proximal part representing the less advanced keratinization. Considered from these findings, it is suggested that the difference in the width of microridges in an important marker of the degree of keratinization. Further, as the surface of the epithelium in the distal part was traced distally the typical microridges became indistinct, and near the anal margin the surface showed a relatively smooth appearance instead of clear microridges. It is suggested that the epithelium near the anal margin represents a high degree of keratinization.

\section{References}

1) Baba E. An electronmicroscopic study of the cpidermis of the transitional skin in the human anal canal. J Jpn Soc Colo-Proctol 1968; 21:3-41. 
2) Brody I. The ultrastructure of the tonofibrils in the keratinization process of normal human epidermis. $J$ Ultrastruct Res 1960; 4:264-297.

3) Cleaton-Jones $P$. Surface characteristics of cells from different layers of keratinized and non-keratinized oral epithelia. J Periodont Res 1975; 10:79-87.

4) Farbman AI. The dual pattern of keratinization in filiform papillae on rat tongue. J Anat 1970; 106:233-242.

5) Fenger C. Anal canal. In Sternberg S (ed.) Histology for Pathologists. New York. Raven Press 1992: pp. 607-623.

6) Fenger $C$ and Knoth $M$. The anal transitional zone. A scanning and transmission electron microscopic investigation of the surface epithelium. Ultrast Pathol 1981; 2:163-173.

7) Fujita $\mathrm{H}$ and Fujita T. Textbook of Histology. Part 2. Tokyo. Igaku Shoin (in Japanese) 1992: p. 142.

8) Goligher JC, Leacock AG and Brossy JJ. The surgical anatomy of the anal canal. Br J Surg 1955; 43:51-61.

9) Henrich M. Clinical topography of the proctodeum. Acta Anat 1980; 106:161-170.

10) Inoue $T$ and Osatake $H$. A new drying method of biological specimens for scanning electron microscopy. The t-butyl alcohol freeze-drying method. Arch Histol Cytol 1988; 51:53-59.

11) Jit I. Anatomy of the rectum and anal canal of the rhesus monkey (Macaca mulatta). J Anat 1974; 117:271-279.

12) Johnson FP. The development of the rectum in human embryo. Am J Anat 1914; 16:1-57.

13) Klotz RG, Pamukcoglu T and Souilliard DH. Transitional coloacogenic carcinoma of the anal canal. Cancer 1967; 20:1727-1745.

14) McMillan MD. The surface structure of the completely and incompletely orthokeratinized oral epithelium in the rat. A light, scanning and transmission electron microscope study. Am J Anat 1979; 156:337-352.

15) Muto $H$. Development of the keratohyalin granules in the esophageal epithelium (in Japanese with English abstract). Acta Anat Nippon 1970; 45:141-148.

16) Nair PNR and Rossinsky K. Crypt architecture of tonsilla lingualis in the monkey, Macaca fascicularis. Cell Tiss Res 1984; 237:619-627.

17) Nakano $T$. Ultrastructural studies of the transitional zone in the nasopharyngeal epithelium, with special reference to the keratinizing process in the mouse. Acta Anat 1986; 127:2247.

18) Nakano $T$. Three-dimensional observations on the penile papillae of the mouse glans. Ann Anat 1996; in press.

19) Nakano $T$ and Muto $H$. The surface structures of the laryngopharyngeal epithelium of the mouse with special reference to the degree of keratinization. Okajimas Folia Anat Jpn 1986; 63:193-208.

20) Nakano $T$ and Muto $H$. Scanning electron microscopic observations on the epithelium lining the mouse anorectal region. Okajimas Folia Anat Jpn 1987; 64:203-222.

21) Patrizi $G$ and Munger $L$. The maturation of cortical keratin in filiform hairs of the rat penis. J Ultrastruct Res 1966; 14:329-344.

22) Rothman S. Physiology and biochemistry of the skin. Chicago. Chicago Univ Press 1954.

23) Symington J. The rectum and anus. J Anat Physiol 1888; 23:106-115.

24) Takano $M$. Term of the anal canal and its measurement (in Japanese). J Jpn Soc Colo-Proctol 1978; 31:226-229. 

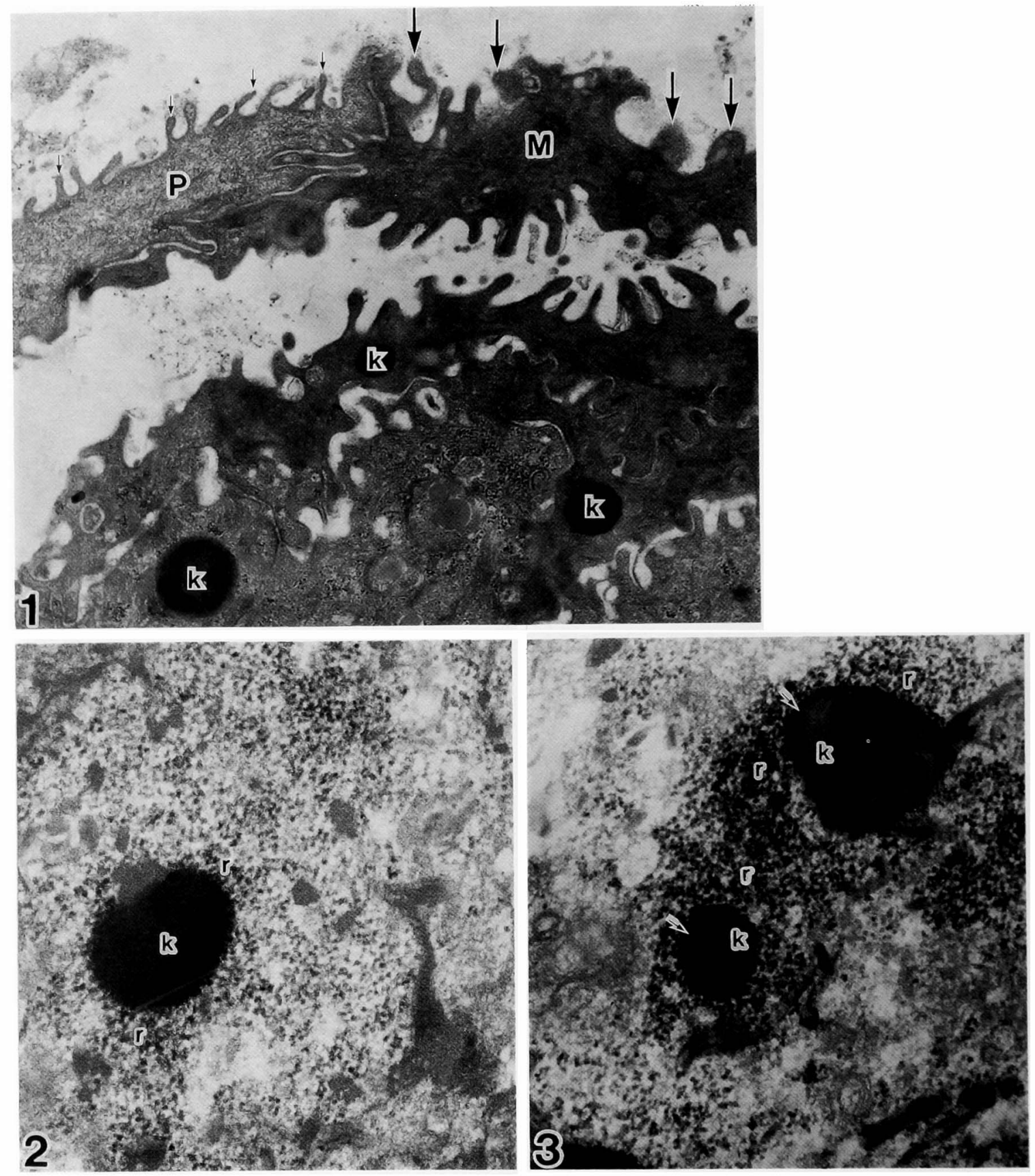

\section{Explanation of Figures}

\section{Plate I}

Fig. 1. Transmission electron micrograph showing the proximal part and the middle one of the anal canal. Round shaped keratohyalin granules (k) are seen in the middle layer. The cytoplasmic filaments in the superficial cells show some tendency to form fibrils in the proximal part $(\mathrm{P})$, while they are embedded in a less opaque substance in the middle part $(\mathrm{M})$. The microridges in the middle part (large arrows) are thicker than those in the proximal part (small arrows). $\times 10,000$

Fig. 2. Higher magnification of the keratohyalin granule $(\mathrm{k})$ in the proximal part. It is surrounded by many ribosomes $(\mathrm{r}) . \times 20,000$

Fig. 3. Higher magnification of the keratohyalin granule (k) in the middle part. It shows a mottled appearance and has a dense rim (arrows). $r=$ ribosome. $\times 20,000$ 
Plate II
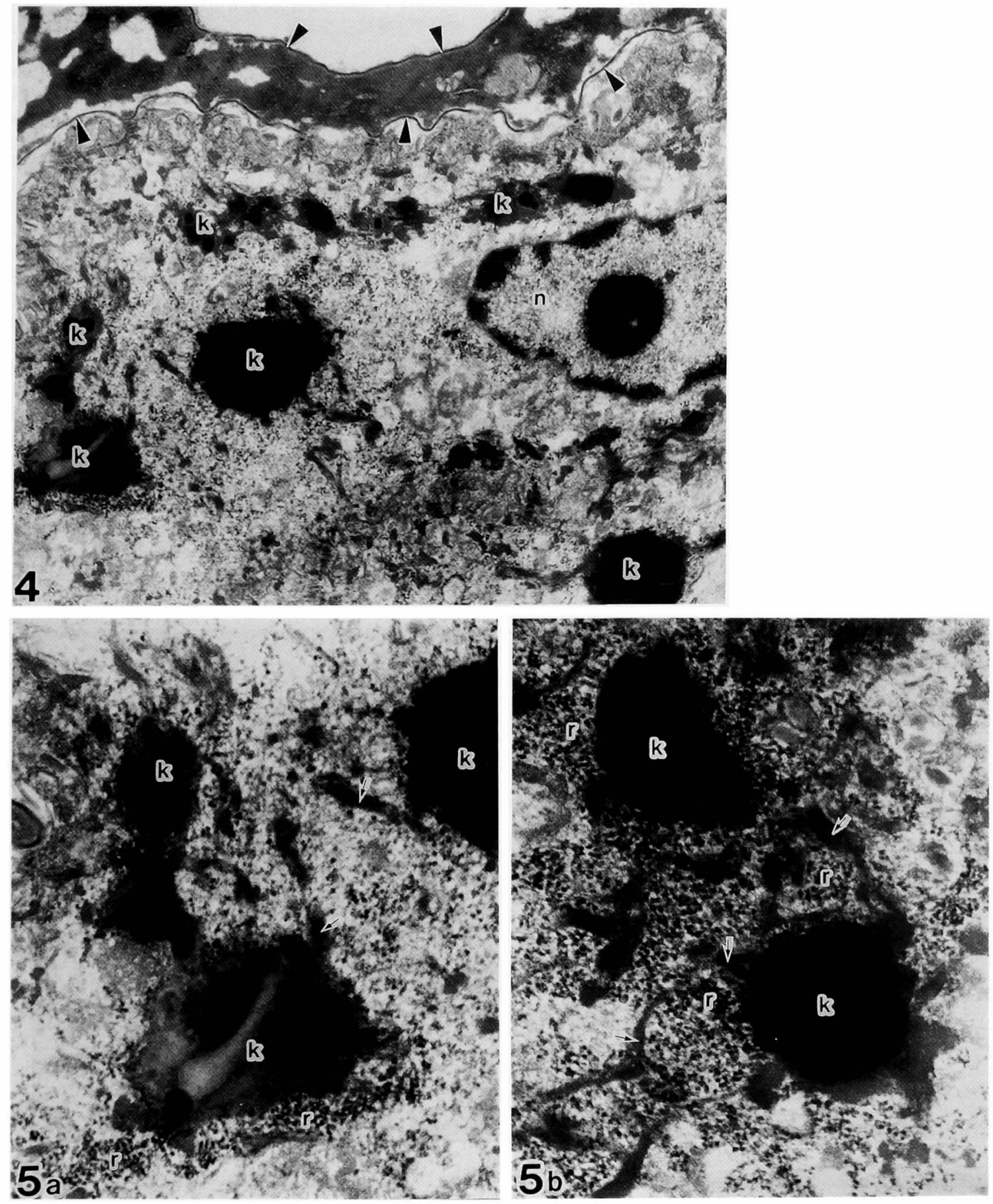

\section{Plate II}

Fig. 4. Transmission electron micrograph showing the distal part of the anal canal. Many keratohyalin granules (k) are seen in the granular layer. The cytoplasmic membrane in the superficial cell consists is thick and dense (arrowheads). $\mathrm{n}=$ nucleus. $\times 10,000$

Fig. 5. Higher magnification of keratohyalin granules (k) in the distal part.

a: Granules with a mottled appearance.

b: Granules with homogeneous contents.

All of the granules are associated with filaments (arrows) and surrounded by ribosomes $(\mathrm{r}) . \times 20,000$ 

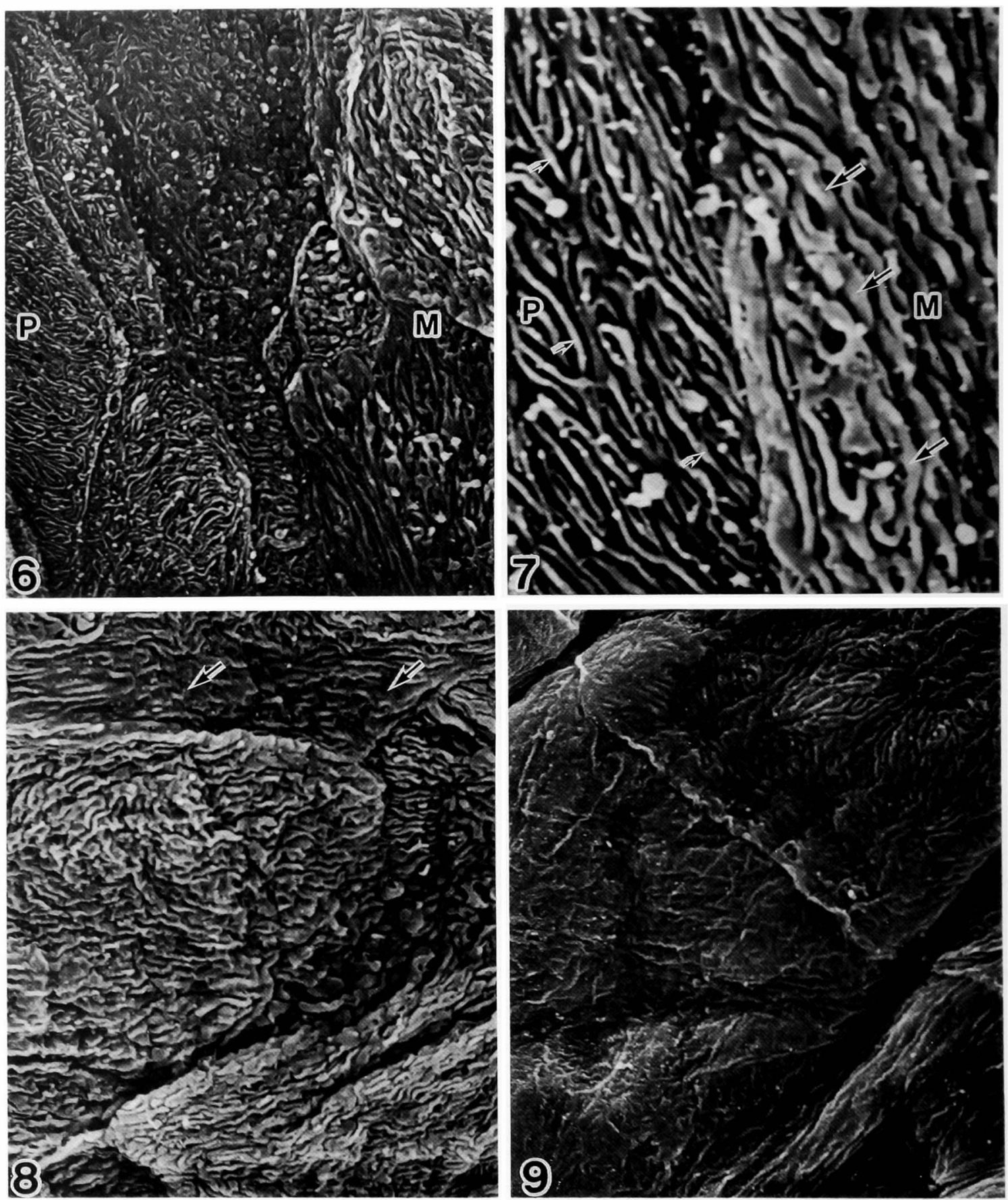

Plate III

Fig. 6. Scanning electron micrograph showing the epithelial surface of the proximal part (P) and the middle one (M) of the anal canal. The surface has a paving-stone appearance. $\times 3,000$

Fig. 7. Higher magnification showing the boundary between the proximal part (P) and the middle one (M). The width of microridges (large arrows) in the middle part is about double that (small arrows) in the proximal part. $\times 10,000$

Fig. 8. The epithelial surface in the distal part. In some sites, the microridges tend to coalesce (arrows). $\times 3,000$

Fig. 9. The epithelial surface near the anal margin. The microridges are not so distinct. $\times 3,000$ 